\title{
Report from the NIST-MAS Workshop on "The Accuracy Barrier in Quantitative EPMA and the Role of Standards"
}

\author{
Dale Newbury, Ryna Marinenko, John Armstrong, John Small, and Eric Steel \\ National Institute of Standards and Technology, Gaithersburg, MD 20899-8371
}

The National Institute of Standards and Technology (NIST) and the Microbeam Analysis Society (MAS) co-sponsored a workshop on the topic of "The Accuracy Barrier in Quantitative EPMA and the Role of Standards" that was held at NIST on April 8-11, 2002. The principal purpose of this workshop was to bring together experts to discuss the electron probe x-ray microanalysis (EPMA) measurement process and to identify factors that limit EPMA accuracy to a practical barrier of approximately $2 \%$ relative. Papers presented at this workshop have now been published as a special issue of the NIST Journal of Research and are also available via the Web.[1] This report will present the highlights of the workshop and serve as a first draft of a roadmap for improving the accuracy of EPMA. Indeed, the use of the word "accuracy" itself is now considered archaic. "Accuracy" is described by the American National Standards Institute (ANSI) as a "qualitative term that describes the closeness between the measured and the true value."[2] "Accuracy" in the measurement process is better represented as a series of error contributions arising from various sources that contribute to an overall uncertainty budget. By identifying the major and minor factors in the uncertainty budget, an effective roadmap can be developed for future improvements to the measurement process by emphasizing those factors where the largest gains can be made.

The workshop topics can be divided into four areas: (1) the EPMA measurement process, including the electron beam instrumentation and the energy dispersive and wavelength dispersive x-ray spectrometry (WDS, EDS); (2) the matrix correction process for the ideal case of a flat, bulk, spatially homogeneous specimen; (3) the impact of standards on the measurement process; and (4) extension of quantitative EPMA into application areas that push the envelope of performance. (1) The EPMA measurement process: The beam energy and current stabilities of modern EPMA columns are such that the error contributions to the intensity measurement should be at the level of $0.1 \%$ or less for practical integration times of $10 \mathrm{~s}$ to $1000 \mathrm{~s}$. Using a Faraday cup to sample the beam current between x-ray measurements can effectively reduce the drift error even when instabilities occur. However, sources of significant error were identified in the signal processing chain for both EDS and WDS spectral measurements. The analyst should determine if the EDS deadtime correction is linear over the operational range (e.g., at least up to 50\% deadtime) by conducting a careful measurement against calibrated beam current. Alternatively, measuring wellresolved peak ratios, e.g., Fe K $\alpha-\mathrm{K} \beta$ and Ta $\mathrm{L} \alpha-\mathrm{L} \beta$, as a function of count rate can provide a test independent of the beam current calibration. For WDS pulse processing, some digital processing systems show significant non-linearity at both high and low count rates.

(2) The matrix correction process: Unless the sample and standard are very close in composition, the accuracy of concentration determination by quantitative EPMA analysis is very dependent on the matrix correction procedure employed. Many matrix correction programs are in current use by the EPMA community, even for the ideal case of flat, bulk, compositionally homogeneous specimens. The programs differ both in the equations employed to correct for compositional effects on electron retardation and scattering, x-ray absorption, and secondary x-ray fluorescence, and the 
fixed parameters used in these equations (e.g., mass absorption coefficients, ionization cross sections). The variation in the results obtained by processing the same experimental data through the different combinations of correction procedures and correction parameters typically ranges from $5 \%$ to $10 \%$ relative and, when the matrix corrections are large as in the case of soft-x-ray analysis, often exceed $20 \%$. Unless the particular correction procedure employed by a laboratory has been tested on a series of secondary standards similar in composition to the unknowns being analyzed and has a known level of accuracy, the uncertainty estimates reported for the analysis should include the range of variation obtained by processing the data through the different common correction programs. The EPMA community should seek multi-laboratory measurements of well characterized secondary standards under carefully controlled analytical conditions to obtain a superior set of experimental k-factors that can be used to better evaluate the accuracy of the matrix corrections and correction parameters for key systems of analytical interest.

(3) Standards: Good microanalysis reference standards play a critical role in the reduction of uncertainty factors leading to improved accuracy in quantitative EPMA. The proper selection of standards, mounting, and surface preparation relative to the unknown specimen is quite important. While simple pure elements and stoichiometric compounds are suitable as standards for many analyses, there is still a considerable need for specific microanalysis reference standards in critical areas such as the aerospace industry (e.g., TiN) and semiconductor industry (e.g., AlGaAs, SiGe). The newly developed NIST Interactive Materials Program will draw more research materials (RM) into the community for cooperative research through a web data base that can be accessed by all laboratories studying them, with eventual elevation to standard reference material (SRM) status. Many of the RMs described at the workshop, such as the Corning glasses, the rare-earth element standards, and the zirconites, are excellent candidates for the NIST Interactive Materials Program. (4) Extreme measurement situations at the limits: Many critical applications of x-ray microanalysis involve measurement situations at the edge of the operational envelope. (a) Particles and rough samples: The geometric factors of size and shape modify the generation and propagation of x-rays, creating a complex spatial response function when compared to a flat, bulk standard. While various correction schemes exist, a strategy that minimizes the uncertainty budget is to operate at the lowest possible beam energy to return as closely as possible to the bulk case. Contamination must be minimized for this approach to be successful. (b) Low voltage microanalysis: High lateral and shallow depth resolutions are possible with $\mathrm{E}_{0}<5 \mathrm{keV}$. However, serious limitations arise with conventional Si-EDS for low photon energy spectrometry because of poor spectral resolution and low fluorescence yield for the L- and M-shell x-rays that must be used instead of the K- and Lshells usually chosen. WDS is too inefficient for low beam current field emission SEMs, but x-ray optic augmented WDS and microcalorimetry hold promise for future progress. (c) VP-SEM and ESEM enable novel operating conditions and unique experiments, but the lateral resolution of $\mathrm{x}$-ray microanalysis of bulk specimens is inevitably compromised by gas scattering. Useful analysis can be performed for dispersed single particles providing careful attention is paid to the analytical blank.

\section{References}

1.Special issue of J. Res. NIST, v107/No. 6 (2002-EDS, low photon energy x-ray spectrometry becomes problematic for many important appli); ISSN 1044p; all papers available as .pdf files at htttp://www.nist.gov/jres

2. American National Standard for Calibration-U.S. Guide to the Expression of Uncertainty in Measurement, B.2.14, NCSL International, Boulder, Colorado, ANSI/NCSL Z540-2-1997 